\title{
Metric products and continuation of isotone functions
}

\author{
O. Dovgoshey, E. Petrov and G. Kozub
}

\begin{abstract}
Let $\mathbb{R}_{+}=[0, \infty)$ and let $A \subseteq \mathbb{R}_{+}^{n}$. We have found the necessary and sufficient conditions under which a function $\Phi: A \rightarrow \mathbb{R}_{+}$has an isotone subadditive continuation on $\mathbb{R}_{+}^{n}$. It allows us to describe the metrics, defined on the Cartesian product $X_{1} \times \cdots \times X_{n}$ of given metric spaces $\left(X_{1}, d_{X_{1}}\right), \ldots,\left(X_{n}, \ldots, d_{X_{n}}\right)$, generated by the isotone metric preserving functions on $\mathbb{R}_{+}^{n}$. It also shows that the isotone metric preserving functions $\Phi: \mathbb{R}_{+}^{n} \rightarrow \mathbb{R}_{+}$coincide with the first moduli of continuity of the nonconstant bornologous functions $g: \mathbb{R}_{+}^{n} \rightarrow \mathbb{R}_{+}$. We discuss some algebraic properties of sets $X \subseteq \mathbb{R}$ providing the existence of isometric embeddings $f: B \rightarrow X$ for every three-point $B \subseteq \mathbb{R}$. In particular, we prove that every finite subset of $\mathbb{R}$ is isometric to some subset of transcendental real numbers.
\end{abstract}

Mathematics Subject Classification (2010): Primary 54E35, Secondary 26A15, 26B25.

Key words: metric product, isotone metric preserving function of several variables, bornologous function, isotone subadditive function, modulus of continuity.

\section{Introduction}

Let $n$ be a positive integer number.

Definition 1.1. Let $\left(X_{1}, d_{X_{1}}\right), \ldots,\left(X_{n}, d_{X_{n}}\right)$ be metric spaces and let $P=X_{1} \times \cdots \times X_{n}$ be the Cartesian product of $X_{1}, \ldots, X_{n}$. A metric d on $P$ is a metric product if there exists a function $\Phi: \mathbb{R}_{+}^{n} \rightarrow \mathbb{R}_{+}$such that the equality

$$
d\left(\left(x_{1}, \ldots, x_{n}\right),\left(y_{1}, \ldots, y_{n}\right)\right)=\Phi\left(d_{X_{1}}\left(x_{1}, y_{1}\right), \ldots, d_{X_{n}}\left(x_{n}, y_{n}\right)\right)
$$

holds for all $\left(x_{1}, \ldots, x_{n}\right),\left(y_{1}, \ldots, y_{n}\right) \in P$. 
Definition 1.2. A function $\Phi: \mathbb{R}_{+}^{n} \rightarrow \mathbb{R}_{+}$is a metric preserving function (of $n$ variables) if $\Phi\left(d_{X_{1}}(\cdot, \cdot), \ldots, d_{X_{n}}(\cdot, \cdot)\right)$ is a metric on $X_{1} \times \cdots \times X_{n}$ for every collection of metric spaces $\left(X_{1}, d_{X_{1}}\right), \ldots,\left(X_{n}, d_{X_{n}}\right)$.

There were published in recent decades a lot of works dealing with metric products. The Euclidean and the Minkowski ranks for arbitrary metric spaces and their behavior with respect to products were studied in [1] and [2]. The papers [3], [4] and [5] concern the long-standing problem of S. Ulam on the uniqueness of decomposition of metric spaces into metric products of indecomposable factors. The search of conditions for multiplicativity of metric properties in terms of properties of the functions $\Phi$ is another natural problem arising under studies of metric products (see, for example, [6], [7], 8], and [9] for some results in this direction). The metric products of an arbitrary infinite or finite family of metric spaces were studied in [10].

In the case where the number of factors in the Cartesian product equals one we obtain a new metric $\Phi \circ d_{1}$. The metrics of such form occupy a special position among metric products (see [11], [12], [13] and [14] for the surveys on the metric preserving functions of one variable).

The present paper deals with the isotone metric preserving functions and isotone metric products. In particular we characterize the metrics which are isotone, amenable or subadditive metric products (see section 3 for the exact definitions and formulations of results). These characterizations are based on the construction of continuation of isotone amenable or isotone subadditive functions defined on subsets of $\mathbb{R}_{+}^{n}$ (see section 2 which play an auxiliary role in the paper).

In section 4 we prove that the isotone metric preserving functions coincide with the moduli of continuity of nonconstant bornologous functions $f: \mathbb{R}_{+}^{n} \rightarrow \mathbb{R}_{+}$. It is a generalization of similar one-dimensional result from [17]. The fifth final section of the paper has the origin in an interesting observation of I. Herbut and M. Moszyǹska that to establish some condition of multiplicativity it often suffices to examine the metric products on $\mathbb{R}^{2} \times \mathbb{R}^{2}$ with the usual Euclidean metrics in the factors (see [7]). The main reason of this phenomenon is the following universal property: for every metric triangle, there is an isometric embedding in $\mathbb{R}^{2}$. The metric spaces which are favorable for isometric embeddings of the triangles situated in $\mathbb{R}=(-\infty, \infty)$ play a similar role for metric preserving, isotone, functions (see Theorem 5.1). Examples of such spaces can be found in section 5 . 


\section{Continuation of isotone functions}

Let $\bar{x}=\left(x_{1}, \ldots, x_{n}\right), \bar{y}=\left(y_{1}, \ldots, y_{n}\right)$ be two vectors from $\mathbb{R}_{+}^{n}$. Then we write:

(i) $\bar{x} \leqslant \bar{y} \Leftrightarrow x_{i} \leqslant y_{i}$ for every $i \in\{1, \ldots, n\}$;

(ii) $\bar{x}<\bar{y} \Leftrightarrow \bar{x} \leqslant \bar{y}$ and $\bar{x} \neq \bar{y}$;

(iii) $|\bar{x}-\bar{y}|:=\left(\left|x_{1}-y_{1}\right|, \ldots,\left|x_{n}-y_{n}\right|\right)$.

We need the following definition.

Definition 2.1. Let $A \subseteq \mathbb{R}_{+}^{n}$. A function $f: A \rightarrow \mathbb{R}_{+}$is isotone if the implication

$$
(\bar{x} \leqslant \bar{y}) \Rightarrow(f(\bar{x}) \leqslant f(\bar{y}))
$$

holds for all $\bar{x}, \bar{y} \in A$.

It is easy to see that $f: \mathbb{R}_{+}^{n} \rightarrow \mathbb{R}_{+}$is isotone if and only if $f$ is increasing by coordinates, i.e., $f$ is separately increasing in every variable.

For $\bar{a} \in \mathbb{R}_{+}^{n}$ we denote by $\bar{a}^{\nabla}$ the lower cone of $\bar{a}$ in the partially ordered set $\left(\mathbb{R}_{+}^{n}, \leqslant\right)$, i.e.,

$$
\bar{a}^{\nabla}=\left\{\bar{x} \in \mathbb{R}_{+}^{n}: \bar{x} \leqslant \bar{a}\right\} .
$$

The following lemma gives us the necessary and sufficient conditions under which a "partially defined" isotone function is a restriction of a "completely defined" isotone function.

Lemma 2.2. Let $A$ be a subset of $\mathbb{R}_{+}^{n}$ and let $\Phi: A \rightarrow \mathbb{R}_{+}$be an isotone function. The following conditions are equivalent.

(i) There is an isotone function $\Psi: \mathbb{R}_{+}^{n} \rightarrow \mathbb{R}_{+}$such that

$$
\left.\Psi\right|_{A}=\Phi
$$

where $\left.\Psi\right|_{A}$ is the restriction of $\Psi$ on the set $A$.

(ii) The inequality

$$
\sup \left\{\Phi(\bar{x}): \bar{x} \in A \cap \bar{b}^{\nabla}\right\}<\infty
$$

holds for every $\bar{b} \in \mathbb{R}_{+}^{n}$.

Proof. The implication (i) $\Rightarrow$ (ii) is trivial. Suppose that condition (ii) is fulfilled. Let us define

$$
\Phi^{*}(\bar{y}):=\sup \left\{\Phi(\bar{x}): \bar{x} \in A \cap \bar{y}^{\nabla}\right\}, \quad \bar{y} \in \mathbb{R}_{+}^{n} .
$$


Inequality (2.3) implies the double inequality

$$
0 \leqslant \Phi^{*}(\bar{a})<\infty
$$

for every $\bar{a} \in \mathbb{R}_{+}^{n}$. (In particular we take $\Phi(\bar{a})=\sup \varnothing=0$ if $\bar{a}^{\nabla} \cap A=\varnothing$.) Hence $\Phi^{*}$ is a nonnegative function on $\mathbb{R}_{+}^{n}$. Since the inclusion $\bar{a}^{\nabla} \cap A \subseteq$ $\bar{b}^{\nabla} \cap A$ holds for $\bar{a} \leqslant \bar{b}$, the function $\Phi^{*}$ is isotone. Definition 2.1 and (2.4) imply $\Phi(\bar{a})=\Phi^{*}(\bar{a})$ for $\bar{a} \in A$. Consequently (2.2) holds with $\Psi=\Phi^{*}$. Condition (i) follows.

Remark 2.3. It is easy to prove the inequality

$$
\Psi(\bar{x}) \geqslant \Phi^{*}(\bar{x})
$$

for every $\bar{x} \in \mathbb{R}_{+}^{n}$ and every isotone function $\Psi: \mathbb{R}_{+}^{n} \rightarrow \mathbb{R}_{+}$satisfying (2.2).

Definition 2.4. Let $A \subseteq \mathbb{R}_{+}^{n}$ and let $\overline{0}=(0, \ldots, 0) \in A$. We shall say that a function $\Phi: A \rightarrow \mathbb{R}_{+}^{n}$ is amenable if $\Phi(\overline{0})=0$ and $\Phi(\bar{x})>0$ for every $\bar{x} \in A \backslash\{\overline{0}\}$.

For $n=1$ and $A=\mathbb{R}_{+}$, Definition 2.4 turns into the definition of amenable functions from [15].

Let $t \in(0, \infty)$ and $j_{0} \in\{1, \ldots, n\}$. Define the vectors $\bar{t}^{j_{0}}=\left(t_{1}^{j_{0}}, \ldots, t_{n}^{j_{0}}\right) \in$ $\mathbb{R}_{+}^{n}$ by the rule

$$
t_{i}^{j_{0}}:= \begin{cases}t & \text { if } i=j_{0}, \\ 0 & \text { if } i \neq j_{0} .\end{cases}
$$

Lemma 2.5. Let $A$ be a subset of $\mathbb{R}_{+}^{n}, \overline{0} \in A$ and let $\Phi: A \rightarrow \mathbb{R}_{+}$be an isotone amenable function. The function $\Phi^{*}: \mathbb{R}_{+}^{n} \rightarrow \mathbb{R}_{+}$defined by 2.4 is amenable if and only if for every $j_{0} \in\{1, \ldots, n\}$ and $\varepsilon \in(0, \infty)$ there is $t=t\left(\varepsilon, j_{0}\right) \in(0, \infty)$ such that

$$
t \leqslant \varepsilon \text { and } \bar{t}^{j_{0}} \in A \text {. }
$$

Proof. Suppose that for every $j_{0} \in\{1, \ldots, n\}$ and $\varepsilon \in(0, \infty)$ there is $t=t\left(\varepsilon, j_{0}\right) \in(0, \infty)$ such that (2.7) holds. It was shown in the proof of Lemma 2.2 that $\Phi^{*}(\overline{0})=\Phi(\overline{0})=0$ and $0 \leqslant \Phi^{*}(\bar{x})<\infty$ for $\bar{x} \in \mathbb{R}_{+}^{n}$. Consequently $\Phi^{*}$ is amenable if and only if

$$
\Phi(\bar{y})>0
$$

for $\bar{y}>\overline{0}$. Now we prove inequality (2.8). By (2.1) the inequality $\bar{y}>\overline{0}$ holds if and only if there are $\varepsilon>0$ and $j_{0} \in\{1, \ldots, n\}$ such that $y_{j_{0}} \geqslant \varepsilon$. By the supposition there is $\bar{t}^{j_{0}} \in A$ for which

$$
\overline{0}<\bar{t}^{j_{0}} \leqslant \bar{y} .
$$


Consequently $\bar{t}^{j_{0}} \in A \cap \bar{y}^{\nabla}$. Hence

$$
\Phi^{*}(\bar{y})=\sup \left\{\Phi(\bar{x}): \bar{x} \in A \cap \bar{y}^{\nabla}\right\} \geqslant \Phi\left(\bar{t}^{j_{0}}\right)
$$

Since $\Phi$ is amenable and $\bar{t}^{j_{0}}>\overline{0}$ we have $\Phi\left(\bar{t}^{j_{0}}\right)>0$. Inequality (2.8) follows.

Conversely, suppose that $\Phi^{*}(y)$ is amenable but there is $j_{0} \in\{1, \ldots, n\}$ and $\varepsilon>0$ such that

$$
\bar{t}^{j_{0}} \notin A
$$

for every $t \in(0, \varepsilon]$. Then $A \cap \bar{\varepsilon}^{j_{0} \nabla}=\{\overline{0}\}$, so that

$$
\Phi^{*}\left(\bar{\varepsilon}^{j}\right)=\sup \{\Phi(\bar{x}): \bar{x} \in\{\overline{0}\}\}=\Phi(\overline{0})=0 .
$$

Hence $\Phi^{*}$ is not amenable, contrary to the supposition.

Remark 2.6. The conclusion of Lemma 2.5 can be reformulated by the following way.

- The function $\Phi^{*}: A \rightarrow \mathbb{R}_{+}$is amenable if and only if $A-\{\overline{0}\}$ is a coinitial subset of $\mathbb{R}_{+}^{n}-\{\overline{0}\}$.

For every $\bar{a}=\left(a_{1}, \ldots, a_{n}\right) \in \mathbb{R}_{+}^{n}$ and $j \in\{1, \ldots, n\}$ define $\operatorname{pr}_{j}(\bar{a})=a_{j}$ and denote by $\bar{a}^{\Delta}$ the upper cone of $\bar{a}$, i.e.,

$$
\bar{a}^{\Delta}=\left\{\bar{x} \in \mathbb{R}_{+}^{n}: \bar{x} \geqslant \bar{a}\right\} .
$$

Lemma 2.7. Let $A$ be a subset of $\mathbb{R}_{+}^{n}, \overline{0} \in A$ and let $\Phi: A \rightarrow \mathbb{R}_{+}$be an isotone and amenable. The following conditions are equivalent

(i) There is an isotone amenable function $\Psi: \mathbb{R}_{+}^{n} \rightarrow \mathbb{R}_{+}$such that

$$
\left.\Psi\right|_{A}=\Phi .
$$

(ii) We have inequality (2.3) for every $\bar{b} \in \mathbb{R}_{+}^{n}$ and the equalities

$$
\inf \left(\operatorname{pr}_{j}(B)\right)=0, \quad j=1, \ldots, n,
$$

hold for every $B \subseteq A$ with $\inf (\Phi(B))=0$.

Proof. (i) $\Rightarrow$ (ii) Suppose (i) holds. By Lemma 2.2 inequality (2.3) holds for every $\bar{b} \in \mathbb{R}_{+}^{n}$. Assume that there are $B \subseteq A$ and $j_{0} \in\{1, \ldots, n\}$ with

$$
\inf (\Phi(B))=0
$$

and

$$
t=\inf \left(\operatorname{pr}_{j}(B)\right)>0
$$


From (2.9) and (2.10) the inequality $t>\infty$ follows. Indeed, if $t=+\infty$, then $B=\varnothing$, so that $\inf (\Phi(B))=+\infty$, contrary to (2.9). Let $\bar{t}^{j_{0}}$ be the vector defined by (2.6) . Then the inequality $\bar{t}^{j_{0}} \leqslant \bar{b}$ holds for every $\bar{b} \in B$. Since $\Psi$ is amenable and isotone and $\bar{t}^{j_{0}}>\overline{0}$, we obtain

$$
0<\Psi\left(\bar{t}^{j_{0}}\right) \leqslant \inf (\Psi(B))=\inf (\Phi(B)),
$$

contrary to (2.9). This contradiction shows that the implication (i) $\Rightarrow$ (ii) is true.

(ii) $\Rightarrow$ (i) Suppose that condition (ii) is fulfilled. Consider first the case when for every $j_{0} \in\{1, \ldots, n\}$ there is $\bar{x} \in A$ such that

$$
\operatorname{pr}_{j_{0}}(\bar{x})>0
$$

Write

$$
\tau_{j}:=\sup \left(\operatorname{pr}_{j} A\right), \quad j \in\{1, \ldots, n\} .
$$

Inequality (2.11) implies that $\tau_{j}>0$ for every $j$. Let us continue $\Phi$ from $A$ to the set

$$
\Gamma=A \cup\left(\bigcup_{j=1}^{n}\left\{\bar{t}^{j}: t \in\left(0, \tau_{j}\right)\right\}\right)
$$

by the rule

$$
\Phi^{*}(\bar{a})=\left\{\begin{array}{l}
\Phi(\bar{a}) \quad \text { if } a \in A \\
\inf \left\{\Phi(\bar{x}): \bar{x} \in A \cap \bar{a}^{\Delta}\right\} \quad \text { if } \bar{a} \in \bigcup_{j=1}^{n}\left\{\bar{t}^{j}: t \in\left(0, \tau_{j}\right)\right\} .
\end{array}\right.
$$

If $\bar{a} \in A \cap\left(\bigcup_{j=1}^{n}\left\{\bar{t}^{j}: t \in\left(0, \tau_{j}\right)\right\}\right)$, then $\bar{a} \in A \cap \bar{a}^{\Delta}$ and, since $\Phi$ is isotone, $\Phi(\bar{x}) \geqslant \Phi(\bar{a})$ for every $\bar{x} \in A \cap \bar{a}^{\Delta}$. Thus we obtain

$$
\Phi^{*}(\bar{a})=\inf \left\{\Phi(\bar{x}): \bar{x} \in A \cap \bar{a}^{\Delta}\right\}=\Phi(a)
$$

for every $\bar{a} \in A \cap\left(\bigcup_{j=1}^{n}\left\{\bar{t}^{j}: t \in\left(0, \tau_{j}\right)\right\}\right)$, i.e., $\Phi^{*}$ is correctly defined. Using the isotonicity of $\Phi$ and the definition of $\Phi^{*}$ we can easily show that $\Phi^{*}(\bar{x})$ is finite for every $\bar{x} \in \Gamma$ and $\Phi^{*}: \Gamma \rightarrow \mathbb{R}_{+}$is isotone. To prove that $\Phi^{*}$ is amenable suppose that there is $\bar{\gamma} \in \Gamma$ such that $\Phi^{*}(\bar{\gamma})=0$. Since $\Phi$ is amenable, the last equality shows

$$
\bar{\gamma} \in \bigcup_{j=1}^{n}\left\{\bar{t}^{j}: t \in\left(0, \tau_{j}\right)\right\}
$$


Consequently

$$
0=\Phi^{*}(\bar{\gamma})=\inf \left\{\Phi(\bar{x}): \bar{x} \in A \cap \bar{\gamma}^{\Delta}\right\}=\inf \left(\Phi\left(A \cap \bar{\gamma}^{\Delta}\right)\right) .
$$

Membership relation (2.14) implies that there are $j_{0} \in\{1, \ldots, n\}$ and $t \in$ $\left(0, \tau_{j_{0}}\right)$ such that $\bar{\gamma}=\bar{t}^{j_{0}}$. Condition (ii) and (2.15) give us the equality

$$
\inf \left(\operatorname{pr}_{j_{0}}\left(A \cap \bar{t}^{j_{0} \Delta}\right)\right)=0 .
$$

In particular there is $\bar{x} \in\left(A \cap \bar{t}^{j_{0} \Delta}\right)$ such that

$$
\operatorname{pr}_{j_{0}}(\bar{x})<t
$$

By definition of the upper cone we have $\operatorname{pr}_{j}(\bar{x}) \geqslant \operatorname{pr}_{j}\left(\bar{t}^{j_{0}}\right)$ for every $\bar{x} \in \bar{t}^{j_{0} \Delta}$ and $j \in\{1, \ldots, n\}$. Consequently $\operatorname{pr}_{j_{0}}(\bar{x}) \geqslant \operatorname{pr}_{j_{0}}\left(\bar{t}^{j_{0}}\right)=t$, contrary to (2.16). Thus $\Phi^{*}$ is amenable. Consider now the case where there is $j \in\{1, \ldots, n\}$ such that $\operatorname{pr}_{j}(A)=\{0\}$. We may assume, after a suitable permutation, that there is $j_{0} \in\{1, \ldots, n\}$ such that

$$
\tau_{j}=\sup \left(\operatorname{pr}_{j}(A)\right)=0
$$

if and only if $1 \leqslant j \leqslant j_{0}$. Let us continue $\Phi$ from $A$ to the set

$$
A_{0}=A \cup\left(\bigcup_{j=1}^{j_{0}}\left\{\bar{t}^{j}: t \in(0, \infty)\right\}\right)
$$

as

$$
\Phi_{0}(\bar{a})=\left\{\begin{array}{l}
\Phi(\bar{a}) \quad \text { if } a \in A \\
t \quad \text { if } \bar{a}=\bar{t}^{j} \text { with } j=\left\{1, \ldots, j_{0}\right\} .
\end{array}\right.
$$

It is easy to prove that $\Phi_{0}: A_{0} \rightarrow \mathbb{R}_{+}$is an isotone and amenable continuation of $\Phi: A \rightarrow \mathbb{R}_{+}$. Now for every $j \in\{1, \ldots, n\}$ there is $\bar{x} \in A_{0}$ such that inequality (2.11) holds. Consequently there is a continuation $\Phi^{*}$ of $\Phi^{0}$ on the set $\Gamma$ (see (2.12) ) defined as in (2.13). To find a continuation of $\Phi^{*}: \Gamma \rightarrow \mathbb{R}_{+}$ to an isotone amenable function $\Psi: \mathbb{R}_{+}^{n} \rightarrow \mathbb{R}_{+}$it suffices to note that for every $j \in\{1, \ldots, n\}$ and every $\varepsilon \in(0, \infty)$ there is $t=t(\varepsilon, j) \in(0, \infty)$ such that $t \leqslant \varepsilon$ and $\bar{t}^{j} \in \Gamma$. Hence a desirable continuation can be obtained as in Lemma 2.5.

Recall that a function $\Phi: \mathbb{R}_{+}^{n} \rightarrow \mathbb{R}_{+}$is subadditive if the inequality

$$
\Phi(\bar{x}+\bar{y}) \leqslant \Phi(\bar{x})+\Phi(\bar{y})
$$

holds for all $\bar{x}, \bar{y} \in \mathbb{R}_{+}^{n}$.

Now we shall give an extension of this property to the case of isotone functions defined on arbitrary subsets of $\mathbb{R}_{+}^{n}$. 
Definition 2.8. Let $A$ be a subset of $\mathbb{R}_{+}^{n}$. An isotone function $\Phi: A \rightarrow \mathbb{R}_{+}$ is subadditive if the implication

$$
\left(\bar{x} \leqslant \sum_{i=1}^{m} \bar{x}^{i}\right) \Rightarrow\left(\Phi(\bar{x}) \leqslant \sum_{i=1}^{m} \Phi\left(\bar{x}^{i}\right)\right)
$$

holds for all $\bar{x}, \bar{x}^{1}, \ldots, \bar{x}^{m} \in A$ and every positive integer number $m \geqslant 2$.

Remark 2.9. It is easy to prove that a function $\Phi: A \rightarrow \mathbb{R}_{+}$is isotone and subadditive if and only if the implication (2.18) holds for every $\bar{x} \in A$ and all $\bar{x}^{1}, \ldots, \bar{x}^{m} \in A$ and every integer number $\mathbf{m} \geqslant \mathbf{1}$.

Remark 2.10. If $\Phi: \mathbb{R}_{+}^{n} \rightarrow \mathbb{R}_{+}$is isotone, then (2.17) holds for all $\bar{x}, \bar{y} \in \mathbb{R}_{+}^{n}$ if and only if (2.18) is true for all $\bar{x}, \bar{x}^{1}, \ldots, \bar{x}^{m} \in \mathbb{R}_{+}^{n}$ with $m \geqslant 2$. Thus Definition 2.8 is an equivalent to the usual definition of subadditivity if $A=\mathbb{R}_{+}^{n}$ and $\Phi$ is isotone.

Lemma 2.11. Let $A$ be a nonempty subset of $\mathbb{R}_{+}^{n}$. The following conditions are equivalent for every function $\Phi: A \rightarrow \mathbb{R}_{+}$.

(i) The function $\Phi$ is isotone and subadditive.

(ii) There is an isotone and subadditive function $\Psi: \mathbb{R}_{+}^{n} \rightarrow \mathbb{R}_{+}$such that $\left.\Psi\right|_{A}=\Phi$.

Proof. The implication (ii) $\Rightarrow$ (i) follows directly from Remark 2.10, Suppose now that $\Phi$ is isotone and subadditive. We shall construct an isotone subadditive function $\Psi: \mathbb{R}_{+}^{n} \rightarrow \mathbb{R}_{+}$such that $\left.\Psi\right|_{A}=\Phi$. For every $\bar{x} \in \mathbb{R}_{+}^{n}$ define the subset $\mathbf{S}(\bar{x})=\mathbf{S}(\bar{x}, A)$ of the set $\bigcup_{k=1}^{\infty} A^{k}$, where $A^{1}=A, A^{2}=A \times A$, $A^{3}=A \times A \times A$ and so on, by the rule

- an element $\left(\bar{x}^{1}, \ldots, \bar{x}^{k}\right)$ of the set $\bigcup_{k=1}^{\infty} A^{k}$ belongs to $\mathbf{S}(\bar{x})$ if and only if

$$
\bar{x} \leqslant \sum_{i=1}^{k} \bar{x}^{i}
$$

First consider the case when $\mathbf{S}(\bar{x}) \neq \varnothing$ for every $\bar{x} \in \mathbb{R}_{+}^{n}$. Define the function $\Psi: \mathbb{R}_{+}^{n} \rightarrow \mathbb{R}_{+}$as

$$
\Psi(\bar{x}):=\inf \left\{\sum_{i=1}^{m} \Phi\left(\bar{x}^{i}\right):\left(\bar{x}^{1}, \ldots, \bar{x}^{m}\right) \in \mathbf{S}(\bar{x})\right\}
$$


(We have $\Psi(\bar{x}) \in \mathbb{R}_{+}$for every $\bar{x} \in \mathbb{R}_{+}^{n}$ because the relation $\mathbf{S}(\bar{x}) \neq \varnothing$ implies the inequality $\Psi(\bar{x})<+\infty$.) Let us show that $\Psi$ gives us the wanted continuation of $\Phi$. Let $\bar{x}, \bar{y} \in \mathbb{R}_{+}^{n}$ and $\bar{y} \leqslant \bar{x}$. The inequality $\bar{y} \leqslant \bar{x}$ and (2.19) imply $\bar{y} \leqslant \sum_{i=1}^{m} \bar{x}^{i}$. Hence we obtain the inclusion $\mathbf{S}(\bar{y}) \supseteq \mathbf{S}(\bar{x})$ for $\bar{y} \leqslant \bar{x}$. Using this inclusion and (2.20) we see that the implication

$$
(\bar{y} \leqslant \bar{x}) \Rightarrow(\Psi(\bar{y}) \leqslant \Psi(\bar{x}))
$$

holds. Thus $\Psi$ is isotone.

To prove the subadditivity of $\Psi$ consider arbitrary $\bar{x}, \bar{y} \in \mathbb{R}_{+}^{n}$. It follows from (2.20) that for every $\varepsilon>0$ there are

$$
\left(\bar{x}^{1}, \ldots, \bar{x}^{m}\right) \in \mathbf{S}(\bar{x}) \text { and }\left(\bar{y}^{1}, \ldots, \bar{y}^{l}\right) \in \mathbf{S}(\bar{y})
$$

such that

$$
\Psi(\bar{x})+\varepsilon \geqslant \sum_{i=1}^{m} \Phi\left(\bar{x}^{i}\right) \text { and } \Psi(\bar{y})+\varepsilon \geqslant \sum_{i=1}^{l} \Phi\left(\bar{y}^{i}\right) .
$$

Let $\bar{z}=\bar{x}+\bar{y}$. Then we have

$$
\bar{z} \leqslant \sum_{i=1}^{m} \bar{x}^{i}+\sum_{i=1}^{l} \bar{y}^{i}
$$

Define $\bar{z}^{i}, i=1, \ldots, m+l$ by the rule:

$$
\bar{z}^{i}= \begin{cases}\bar{x}^{i} & \text { if } 1 \leqslant i \leqslant m \\ \bar{y}^{i-m} & \text { if } m+1 \leqslant i \leqslant m+l .\end{cases}
$$

Inequality (2.22) shows that $\left(\bar{z}^{1}, \ldots, \bar{z}^{m}, \bar{z}^{m+1}, \ldots, \bar{z}^{m+l}\right) \in \mathbf{S}(\bar{z})$. Inequalities (2.21), (2.23) and (2.20) imply

$$
\Psi(\bar{z}) \leqslant \sum_{i=1}^{l+m} \Phi\left(\bar{z}^{i}\right)=\sum_{i=1}^{m} \Phi\left(\bar{x}^{i}\right)+\sum_{i=1}^{l} \Phi\left(\bar{y}^{i}\right) \leqslant \Psi(\bar{x})+\Psi(\bar{y})+2 \varepsilon .
$$

Letting $\varepsilon \rightarrow 0$, we have

$$
\Psi(\bar{z}) \leqslant \Psi(\bar{x})+\Psi(\bar{y})
$$

Thus $\Psi$ is subadditive.

It remains to verify that $\left.\Psi\right|_{A}=\Phi$. Let $\bar{x} \in A$ and $\left(\bar{x}^{1}, \ldots, \bar{x}^{k}\right) \in \mathbf{S}(\bar{x})$. Then, by (2.19), we have $\bar{x} \leqslant \sum_{i=1}^{k} \bar{x}^{i}$. If $k=1$, then $\bar{x} \leqslant \bar{x}^{1}$, so that 
$\Phi(\bar{x}) \leqslant \Phi\left(\bar{x}^{1}\right)$ because $\Phi$ is isotone. If $k \geqslant 2$, then using the subadditivity of $\Phi$ we also have the inequality $\Phi(\bar{x}) \leqslant \sum_{i=1}^{k} \Phi\left(\bar{x}^{i}\right)$. Consequently the last inequality holds for every $\left(\bar{x}^{1}, \ldots, \bar{x}^{k}\right) \in \mathbf{S}(\bar{x})$. The inequality

$$
\Phi(\bar{x}) \leqslant \Psi(\bar{x})
$$

follows. To prove the converse inequality

$$
\Psi(\bar{x}) \leqslant \Phi(\bar{x})
$$

note that the point $\bar{x}^{1}=\bar{x}$, belongs to $\mathbf{S}(\bar{x})$ for every $\bar{x} \in A$. Consequently (2.25) follows from (2.20). Inequalities (2.24) and (2.25) imply that $\Phi(\bar{x})=\Psi(\bar{x})$ for every $\bar{x} \in A$, i.e., $\left.\Psi\right|_{A}=\Phi$.

Consider now the case when there is $\bar{a}=\left(a_{1}, \ldots, a_{n}\right) \in \mathbb{R}_{+}^{n}$ such that $\mathbf{S}(\bar{a})=\varnothing$. This equality and the definition of the set $\mathbf{S}(\bar{x})$ imply that there exists $j_{0} \in\{1, \ldots, n\}$ such that

$$
\forall \bar{y} \in A \quad \operatorname{pr}_{j_{0}}(\bar{y})=0 \text { but } a_{j_{0}}=\operatorname{pr}_{j_{0}}(\bar{a})>0 .
$$

Let us denote by $J_{0}$ the set of all elements $j_{0}$ of $\{1, \ldots, n\}$ satisfying condition (2.26). For every $t \in(0, \infty)$ and $j_{0} \in J_{0}$ write $\bar{t}^{j_{0}}$ for the vector defined by (2.6). Let us continue $\Phi$ from the set $A$ to the set

$$
\Gamma:=A \cup\left(\bigcup_{j \in J_{0}}\left\{\bar{t}^{j}: t \in(0, \infty)\right\}\right)
$$

by the rule

$$
\Phi^{\circ}(\bar{a})= \begin{cases}\Phi(\bar{a}) & \text { if } \bar{a} \in A \\ c & \text { if } \bar{a} \in \Gamma \backslash A .\end{cases}
$$

where $c$ is an arbitrary point from $\mathbb{R}_{+}$. It is easy to see that

$$
\left.\Phi^{\circ}\right|_{A}=\Phi \text { and } \mathbf{S}(\bar{x}, \Gamma) \neq \varnothing
$$

for every $\bar{x} \in \mathbb{R}_{+}^{n}$. Hence if $\Phi^{\circ}$ is isotone and subadditive, then replacing in (2.20) $\Phi$ by $\Phi^{\circ}$ we can finish the proof.

In accordance with Remark 2.9, $\Phi^{\circ}$ is isotone and subadditive if implication (2.18) holds for all $\bar{x}, \bar{x}^{1}, \ldots, \bar{x}^{m} \in \Gamma$ and every $m \geqslant 1$. It is clear that (2.18) holds whenever $\bar{x}=\overline{0}$. To clarify the proof of (2.18) for $\bar{x}>\overline{0}$ first consider the case $m=1$. Note that every nonzero $\bar{x} \in A$ and nonzero $\bar{y} \in \Gamma \backslash A$ are incomparable, i.e., we have neither $\bar{x} \leqslant \bar{y}$ nor $\bar{y} \leqslant \bar{x}$. Consequently if $\overline{0}<\bar{x} \leqslant \bar{x}^{1}$, then either $\bar{x}, \bar{x}^{1} \in A$ or $\bar{x}, \bar{x}^{1} \in \Gamma \backslash A$. The function $\left.\Phi^{\circ}\right|_{A}=\Phi$ is isotone and subadditive by the supposition. The function $\left.\Phi^{\circ}\right|_{\Gamma \backslash A}$ 
is also isotone and subadditive as a constant function. Consequently (2.18) holds when $m=1$, i.e., $\Phi^{\circ}$ is isotone. Let us consider an arbitrary $m \geqslant 1$. Suppose that $\overline{0}<\bar{x} \leqslant \sum_{i=1}^{m} \bar{x}^{i}, \bar{x} \in A$ and $\bar{x}^{1}, \ldots, \bar{x}^{m} \in \Gamma$. Define a subset $I$ of the set $\{1, \ldots, m\}$ by the rule

$$
(i \in I) \Leftrightarrow\left(i \in\{1, \ldots, m\} \text { and } \bar{x}^{i} \in A\right) .
$$

Then, using the definition of the set $\Gamma$, we obtain the inequality $\bar{x} \leqslant \sum_{i \in I} \bar{x}^{i}$. (Note that the implication

$$
\left(\bar{x} \in \Gamma \backslash A \text { and } \overline{0}<\bar{x} \leqslant \sum_{i=1}^{m} \bar{x}^{i}\right) \Rightarrow\left(\bar{x} \leqslant \sum_{i \in I} \bar{x}^{i}\right)
$$

can be considered as a generalization of incomparability of nonzero $\bar{x} \in A$ and $\bar{y} \in \Gamma \backslash A$.) Since $\bar{x}^{i} \in A$ for $i \in I$, the inequality $\bar{x} \leqslant \sum_{i \in I} \bar{x}^{i}$ implies

$$
\Phi^{\circ}(x)=\Phi(\bar{x}) \leqslant \sum_{i \in I} \Phi\left(\bar{x}^{i}\right)=\sum_{i \in I} \Phi^{\circ}\left(\bar{x}^{i}\right) \leqslant \sum_{i=1}^{m} \Phi^{\circ}\left(\bar{x}^{i}\right) .
$$

Similarly we can show the inequality

$$
\Phi^{\circ}\left(\bar{x}^{i}\right) \leqslant \sum_{i=1}^{m} \Phi^{\circ}\left(\bar{x}^{i}\right)
$$

for $\bar{x} \in \Gamma \backslash A$ and $\bar{x}^{1}, \ldots, \bar{x}^{m} \in \Gamma$. Thus (2.18) holds for all $\bar{x}, \bar{x}^{1}, \ldots, \bar{x}^{m} \in$ $\Gamma$.

We finish this section by the following proposition, which is interesting in its own right.

Theorem 2.12. Let $A$ be a subset of $\mathbb{R}_{+}^{n}$ such that for every $j \in\{1, \ldots, n\}$ there is $\bar{a} \in A$ satisfying the inequality $\operatorname{pr}_{j}(\bar{a})>0$. Then, for every $\Phi$ : $A \rightarrow \mathbb{R}_{+}$, the function $\Psi: \mathbb{R}_{+}^{n} \rightarrow \mathbb{R}_{+}$defined by (2.20) has the following properties.

(i) $\Psi$ is isotone and subadditive.

(ii) The inequality $\Phi(\bar{x}) \geqslant \Psi(\bar{x})$ holds for every $\bar{x} \in A$.

(iii) The equality $\Phi=\left.\Psi\right|_{A}$ holds if and only if $\Phi$ is isotone and subadditive. 
(iv) If $F: \mathbb{R}_{+}^{n} \rightarrow \mathbb{R}_{+}$is isotone subadditive function such that the inequality $\Phi(\bar{x}) \geqslant F(\bar{x})$ holds for every $\bar{x} \in A$, then the inequality $\Psi(\bar{x}) \geqslant F(\bar{x})$ also holds for every $\bar{x} \in \mathbb{R}_{+}^{n}$.

This theorem can be proved by modification of the proof of Lemma 2.11 so it can be omitted here.

Remark 2.13. If for given $\Phi: A \rightarrow \mathbb{R}_{+}$there is a function $\Psi: \mathbb{R}_{+}^{n} \rightarrow \mathbb{R}_{+}$ meeting conditions (i)-(iv), then for every $j \in\{1, \ldots, n\}$ there is $\bar{a} \in A$ such that $\operatorname{pr}_{j}(\bar{a})>0$. It can be obtained from (2.28) with $\Phi=\left.\Psi\right|_{A}$.

\section{From metric products to metric preserving functions}

Let us denote by $\mathfrak{F}_{i}^{n}$ the set of isotone metric preserving functions.

Theorem 3.1. Let $f: \mathbb{R}_{+}^{n} \rightarrow \mathbb{R}_{+}$be isotone. Then $f$ belongs to $\mathfrak{F}_{i}^{n}$ if and only if $f$ is subadditive and amenable.

This theorem is a direct consequence of Theorem 2.6 from [10] and Theorem 1 from Chapter 9 of [15].

Definition $3.2([9])$. Let $\left(X_{1}, d_{X_{1}}\right), \ldots,\left(X_{n}, d_{X_{n}}\right)$ be metric spaces. A metric $d$ defined on the product $X_{1} \times \cdots \times X_{n}$ is distance-increasing if

$$
d\left(\left(x_{1}, \ldots, x_{n}\right),\left(y_{1}, \ldots, y_{n}\right)\right) \leqslant d\left(\left(\tilde{x}_{1}, \ldots, \tilde{x}_{n}\right),\left(\tilde{y}_{1}, \ldots, \tilde{y}_{n}\right)\right)
$$

whenever

$$
\left(d_{X_{1}}\left(x_{1}, y_{1}\right), \ldots, d_{X_{n}}\left(x_{n}, y_{n}\right)\right) \leqslant\left(d_{X_{1}}\left(\tilde{x}_{1}, \tilde{y}_{1}\right), \ldots, d_{X_{n}}\left(\tilde{x}_{n}, \tilde{y}_{n}\right)\right) .
$$

Recall that the distance set of a metric space $(X, d)$ is the set

$$
D_{X}=\{d(x, y): x, y \in X\} .
$$

Let $\left(X_{1}, d_{X_{1}}\right), \ldots,\left(X_{n}, d_{X_{n}}\right)$ be metric spaces. We shall say that a metric product $d$ is isotone (subadditive) if there is an isotone (subadditive) function $\Phi: D_{X_{1}} \times \cdots \times D_{X_{n}} \rightarrow \mathbb{R}_{+}$such that (1.1) holds for all $\left(x_{1}, \ldots, x_{n}\right),\left(y_{1}, \ldots, y_{n}\right) \in X_{1} \times \cdots \times X_{n}$.

Lemma 3.3. Let $\left(X_{1}, d_{X_{1}}\right), \ldots,\left(X_{n}, d_{X_{n}}\right)$ be nonempty metric spaces and let $d$ be a metric defined on $P=X_{1} \times \cdots \times X_{n}$. The following conditions are equivalent. 
(i) $d$ is an isotone metric product.

(ii) $d$ is distance-increasing.

Proof. The implication (i) $\Rightarrow$ (ii) is clear. Suppose that $d$ is distanceincreasing. Let us prove that there exists an isotone

$$
\Phi: D_{X_{1}} \times \cdots \times D_{X_{n}} \rightarrow \mathbb{R}_{+}
$$

such that (1.1) holds for all $\left(x_{1}, \ldots, x_{n}\right),\left(y_{1}, \ldots, y_{n}\right) \in P$.

Let us set $d_{X_{i}}\left(x_{i}, y_{i}\right)=d_{X_{i}}\left(\tilde{x}_{i}, \tilde{y}_{i}\right), i=1, \ldots, n$, in Definition 3.2. Then inequality (3.1) implies

$$
d\left(\left(x_{1}, \ldots, x_{n}\right),\left(y_{1}, \ldots, y_{n}\right)\right)=d\left(\left(\tilde{x}_{1}, \ldots, \tilde{x}_{n}\right),\left(\tilde{y}_{1}, \ldots, \tilde{y}_{n}\right)\right) .
$$

Thus, the function $d: P \times P \rightarrow \mathbb{R}_{+}$depends only on the distances $d_{X_{1}}\left(x_{1}, y_{1}\right), \ldots, d_{X_{n}}\left(x_{n}, y_{n}\right)$. Consequently, there exists a function

$$
\Phi: D_{X_{1}} \times \cdots \times D_{X_{n}} \rightarrow \mathbb{R}_{+}^{n}
$$

such that the following diagram

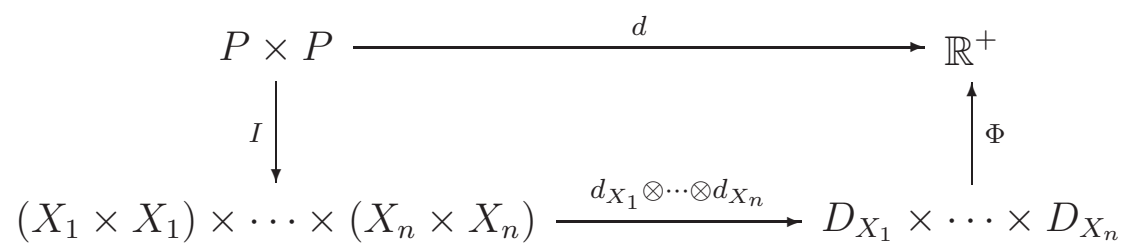

is commutative, where $I$ is the identification mapping,

$$
I\left(\left(x_{1}, \ldots, x_{n}\right),\left(\tilde{x}_{1}, \ldots, \tilde{x}_{n}\right)\right)=\left(\left(x_{1}, \tilde{x}_{1}\right), \ldots,\left(x_{n}, \tilde{x}_{n}\right)\right),
$$

and $d_{X_{1}} \otimes \cdots \otimes d_{X_{n}}$ is the direct product,

$$
d_{X_{1}} \otimes \cdots \otimes d_{X_{n}}\left(\left(x_{1}, \tilde{x}_{1}\right), \ldots,\left(x_{n}, \tilde{x}_{n}\right)\right)=\left(d_{X_{1}}\left(x_{1}, \tilde{x}_{1}\right), \ldots, d_{X_{n}}\left(x_{n}, \tilde{x}_{n}\right)\right) .
$$

Using the fact that $d$ is distance-increasing it is easy to show that $\Phi$ is isotone.

Let us define the metric $\rho_{\infty}$ on $P=X_{1} \times \cdots \times X_{n}$ as

$$
\rho_{\infty}\left(\left(x_{1}, \ldots, x_{n}\right),\left(y_{1}, \ldots, y_{n}\right)\right)=\max _{1 \leqslant i \leqslant n} d_{X_{i}}\left(x_{i}, y_{i}\right) .
$$

We shall say that an isotone metric product $d$ has an isotone (amenable, subadditive) continuation if there is an isotone (amenable, subadditive) function $\Phi: \mathbb{R}_{+}^{n} \rightarrow \mathbb{R}_{+}$such that

$$
d\left(\left(x_{1}, \ldots, x_{n}\right),\left(y_{1}, \ldots, y_{n}\right)\right)=\Phi\left(d_{X_{1}}\left(x_{1}, y_{1}\right), \ldots, d_{X_{n}}\left(x_{n}, y_{n}\right)\right)
$$


for all $\left(x_{1}, \ldots, x_{n}\right),\left(y_{1}, \ldots, y_{n}\right) \in P$.

Let $(X, d)$ and $(Y, \rho)$ be metric spaces. Recall that a mapping $f: X \rightarrow Y$ is bornologous if for every $\varepsilon \in \mathbb{R}_{+}$there is $\delta=\delta(\varepsilon) \in \mathbb{R}_{+}$such that the implication

$$
(d(x, y) \leqslant \varepsilon) \Rightarrow(\rho(f(x), f(y)) \leqslant \delta)
$$

holds for all $x, y \in X$ (see [18, p.6]).

Remark 3.4. It is easy to prove that $f: X \rightarrow Y$ is bornologous if and only if there is an increasing $g: \mathbb{R}_{+} \rightarrow \mathbb{R}_{+}$such that

$$
\rho(f(x), f(y)) \leqslant g(d(x, y))
$$

for all $x, y \in X$.

Theorem 3.5. Let $\left(X_{1}, d_{X_{1}}\right), \ldots,\left(X_{n}, d_{X_{n}}\right)$ be metric spaces. The following conditions are equivalent for every metric d on $P=X_{1} \times \cdots \times X_{n}$.

(i) $d$ is an isotone metric product and has an isotone continuation.

(ii) $d$ is distance-increasing and the identical mapping

$$
P \ni\left(x_{1}, \ldots, x_{n}\right) \mapsto\left(x_{1}, \ldots, x_{n}\right) \in P
$$

is bornologous as a mapping from $\left(P, \rho_{\infty}\right)$ to $(P, d)$.

Proof. (i) $\Rightarrow$ (ii). Let $d$ be an isotone metric product having an isotone continuation. By Lemma 3.3, the metric $d$ is distance-increasing. Let $\Psi$ : $\mathbb{R}_{+}^{n} \rightarrow \mathbb{R}_{+}$be an isotone function such that

$$
d\left(\left(x_{1}, \ldots, x_{n}\right),\left(y_{1}, \ldots, y_{n}\right)\right)=\Psi\left(d_{X_{1}}\left(x_{1}, y_{1}\right), \ldots, d_{X_{n}}\left(x_{n}, y_{n}\right)\right)
$$

for $\left(x_{1}, \ldots, x_{n}\right),\left(y_{1}, \ldots, y_{n}\right) \in P$. Write

$$
A:=D_{X_{1}} \times \cdots \times D_{X_{n}} .
$$

The restriction $\left.\Psi\right|_{A}$ is an isotone function on $A \subseteq \mathbb{R}_{+}^{n}$ and the function $\Psi$ is an isotone continuation of $\left.\Psi\right|_{A}$. Consequently, by Lemma 2.2, the inequality

$$
\sup \left\{\left.\Psi\right|_{A}(\bar{x}): \bar{x} \in A \cap \bar{b}^{\nabla}\right\}<\infty
$$

holds for every $\bar{b} \in \mathbb{R}_{+}^{n}$. Let us take here $\bar{b}=\bar{b}_{\varepsilon}=(\varepsilon, \ldots, \varepsilon)$ with $\varepsilon \in \mathbb{R}_{+}$. We claim that the inequality

$$
d\left(\left(x_{1}, \ldots, x_{n}\right),\left(y_{1}, \ldots, y_{n}\right)\right) \leqslant g\left(\rho_{\infty}\left(\left(x_{1}, \ldots, x_{n}\right),\left(y_{1}, . ., y_{n}\right)\right)\right)
$$


holds for all $\left(x_{1}, . ., x_{n}\right),\left(y_{1}, . ., y_{n}\right) \in P$ with

$$
g(\varepsilon):=\sup \left\{\left.\Psi\right|_{A}(\bar{z}): \bar{z} \in A \cap \bar{b}_{\varepsilon}^{\nabla}\right\}, \quad \varepsilon \in \mathbb{R}_{+} .
$$

Indeed, the relation $\bar{z} \in A \cap \bar{b}_{\varepsilon}^{\nabla}$ holds if and only if there are $\left(x_{1}, \ldots, x_{n}\right)$, $\left(y_{1}, \ldots, y_{n}\right) \in P$ such that $d_{X_{i}}\left(x_{i}, y_{i}\right)=z_{i}, i=1, \ldots, n$, and

$$
\begin{gathered}
\max _{1 \leqslant i \leqslant n} d_{X_{i}}\left(x_{i}, y_{i}\right) \leqslant \varepsilon, \quad \text { i.e }, \\
A \cap \bar{b}_{\varepsilon}^{\nabla}=\left\{d\left(\left(x_{1}, \ldots, x_{n}\right),\left(y_{1}, \ldots, y_{n}\right)\right):\left(x_{1}, \ldots, x_{n}\right),\left(y_{1}, \ldots, y_{n}\right) \in P\right. \\
\text { and } \left.\rho_{\infty}\left(\left(x_{1}, \ldots, x_{n}\right),\left(y_{1}, \ldots, y_{n}\right)\right) \leqslant \varepsilon\right\} .
\end{gathered}
$$

This equality, (3.4) and (3.7) imply (3.6). Since $d$ is distance-increasing, condition (ii) follows (see Remark 2.3).

(ii) $\Rightarrow$ (i). Suppose that (ii) holds. Lemma 3.3 implies that there is an isotone function $\Phi: D_{X_{1}} \times \cdots \times D_{X_{n}} \rightarrow \mathbb{R}_{+}$such that

$$
d\left(\left(x_{1}, \ldots, x_{n}\right),\left(y_{1}, \ldots, y_{n}\right)\right)=\Phi\left(d_{X_{1}}\left(x_{1}, y_{1}\right), \ldots, d_{X_{n}}\left(x_{n}, y_{n}\right)\right)
$$

for all $\left(x_{1}, \ldots, x_{n}\right),\left(y_{1}, \ldots, y_{n}\right) \in P$. To prove that $d$ has an isotone continuation we must find an isotone $\Psi: \mathbb{R}_{+}^{n} \rightarrow \mathbb{R}_{+}$for which

$$
\left.\Psi\right|_{D_{X_{1}} \times \cdots \times D_{X_{n}}}=\Phi \text {. }
$$

To this end it suffices to prove the inequality

$$
\sup \left\{\Phi(\bar{x}): \bar{x} \in\left(D_{X_{1}} \times \cdots \times D_{X_{n}}\right) \cap \bar{b}^{\nabla}\right\}<\infty
$$

for every $\bar{b} \in \mathbb{R}_{+}^{n}$ (see Lemma 2.2). It is clear that $\bar{b} \leqslant \bar{b}_{\varepsilon}$ if $\varepsilon=\max _{1 \leqslant i \leqslant n} b_{i}$. Hence $\left(D_{X_{1}} \times \cdots \times D_{X_{n}}\right) \cap \bar{b}^{\nabla} \subseteq\left(D_{X_{1}} \times \cdots \times D_{X_{n}}\right) \cap \bar{b}_{\varepsilon}^{\nabla}$. Consequently inequality (3.9) follows from

$$
\sup \left\{\Phi(\bar{x}): \bar{x} \in\left(D_{X_{1}} \times \cdots \times D_{X_{n}}\right) \cap \bar{b}_{\varepsilon}^{\nabla}\right\}<\infty .
$$

Since (ii) holds, the last inequality is a consequence of (3.8).

Now we construct a distance-increasing metric which does not have any isotone continuation. For the sake of simplicity, consider a degenerate Cartesian product $P=X_{1} \times \cdots \times X_{n}$ with $n=1$.

Example 3.6. Let $X=[0,1)$ and let $\varphi:[0,1) \rightarrow[0, \infty)$ be a continuous strictly increasing function with $\lim _{t \rightarrow 1} \varphi(t)=\infty$ and $\lim _{t \rightarrow \infty} \varphi(t)=0$. Define a metric $\rho$ on $X$ by the rule

$$
\rho(x, y)=\left\{\begin{array}{l}
\max \{x, y\} \quad \text { if } x \neq y \\
0 \quad \text { if } x=y
\end{array}\right.
$$


It is easy to see that $(X, \rho)$ is an ultrametric space, i.e., the strong triangle inequality

$$
\rho(x, y) \leqslant \max \{\rho(x, z), \rho(z, y)\}
$$

holds for all $x, y, z \in X$. The function $d: X \times X \rightarrow \mathbb{R}_{+}$,

$$
d(x, y)=\varphi(\rho(x, y)), \quad x, y \in X
$$

is also a metric and even an ultrametric on $X$. Note that $d$ is distanceincreasing because $\varphi$ is increasing. We show that the identical mapping $\operatorname{id}(x)=x$ is uniformly continuous but not bornologous if we consider id as a mapping from $(X, \rho)$ to $(X, d)$. The uniform continuity of id follows directly from limit relation $\lim _{t \rightarrow 0} \varphi(t)=0$. If id is bornologous, then, by Remark 2.3 , there is an increasing function $g: \mathbb{R}_{+} \rightarrow \mathbb{R}_{+}$such that $d(x, y)=\varphi(\rho(x, y)) \leqslant g(\rho(x, y))$ for all $x, y \in[0,1)$. Since $g$ is increasing and $\sup _{x, y \in X} \rho(x, y) \leqslant 1$, the inequality $d(x, y) \leqslant g(1)$ holds for all $x, y \in X$. Hence the metric space $(X, d)$ is bounded, contrary to the definition. By Theorem $3.5 d=\varphi \circ \rho$ is a distance-increasing metric which does not have isotone continuations.

Analyzing the previous example we obtain the following conditions under which the isotone metric products have isotone continuations.

Corollary 3.7. Let $\left(X_{1}, d_{X_{1}}\right), \ldots,\left(X_{n}, d_{X_{n}}\right)$ be metric spaces. Suppose that each $\left(X_{i}, d_{X_{i}}\right)$ is either unbounded ar there are $x_{i}, y_{i} \in X_{i}$ such that

$$
d_{X_{i}}\left(x_{i}, y_{i}\right)=\operatorname{diam} X_{i}
$$

where $\operatorname{diam} X_{i}=\sup \left\{d_{X_{i}}(x, y): x, y \in X_{i}\right\}$. Then every isotone metric product on $P=X_{1} \times \cdots \times X_{n}$ has an isotone continuation.

The next theorem gives us an intrinsic description of isotone metric products having isotone amenable continuations.

Theorem 3.8. Let $\left(X_{1}, d_{X_{1}}\right), \ldots,\left(X_{n}, d_{X_{n}}\right)$ be metric spaces. The following conditions are equivalent for every metric $d$ on $P=X_{1} \times \cdots \times X_{n}$.

(i) $d$ is an isotone metric product with an isotone amenable continuation.

(ii) $d$ is distance-increasing and the identical mapping

$$
\operatorname{id}\left(x_{1}, \ldots, x_{n}\right)=\left(x_{1}, \ldots, x_{n}\right), \quad\left(x_{1}, \ldots, x_{n}\right) \in P
$$

is bornologous as a mapping from $\left(P, \rho_{\infty}\right)$ to $(P, d)$ and uniformly continuous as a mapping from $(P, d)$ to $\left(P, \rho_{\infty}\right)$. 
Proof. (i) $\Rightarrow$ (ii) Suppose that condition (i) is fulfilled. Then, in accordance with Theorem 3.5, $d$ is distance-increasing and id is bornologous mapping from $\left(P, \rho_{\infty}\right)$ to $(P, d)$. Thus to prove (ii) it suffices to show that id is an uniformly continuous mapping from $(P, d)$ to $\left(P, \rho_{\infty}\right)$. Suppose the contrary. Then there are $\varepsilon>0$ and two sequences $\left\{\left(x_{1}^{i}, \ldots, x_{n}^{i}\right)\right\}_{i \in \mathbb{N}},\left\{\left(y_{1}^{i}, \ldots, y_{n}^{i}\right)\right\}_{i \in \mathbb{N}}$ such that

$$
\lim _{i \rightarrow \infty} d\left(\left(x_{1}^{i}, \ldots, x_{n}^{i}\right),\left(y_{1}^{i}, \ldots, y_{n}^{i}\right)\right)=0
$$

and

$$
\rho_{\infty}\left(\left(x_{1}^{i}, \ldots, x_{n}^{i}\right),\left(y_{1}^{i}, \ldots, y_{n}^{i}\right)\right) \geqslant \varepsilon
$$

for every $i \in \mathbb{N}$. Passing to subsequences and reordering the factors in $X_{1} \times \cdots \times X_{n}$ we may assume that

$$
d_{X_{1}}\left(x_{1}^{i}, y_{1}^{i}\right) \geqslant \varepsilon, \text { for every } i \in \mathbb{N} .
$$

By condition (i), there is an isotone amenable function $\Phi: \mathbb{R}_{+}^{n} \rightarrow \mathbb{R}_{+}$for which

$$
d\left(\left(x_{1}, \ldots, x_{n}\right),\left(y_{1}, \ldots, y_{n}\right)\right)=\Phi\left(d_{X_{1}}\left(x_{1}, y_{1}\right), \ldots, d_{X_{n}}\left(x_{n}, y_{n}\right)\right)
$$

for all $\left(x_{1}, \ldots, x_{n}\right),\left(y_{1}, \ldots, y_{n}\right) \in P$. Using (3.11) we obtain

$$
\begin{gathered}
d\left(\left(x_{1}^{i}, \ldots, x_{n}^{i}\right),\left(y_{1}^{i}, \ldots, y_{n}^{i}\right)\right)=\Phi\left(d_{X_{1}}\left(x_{1}^{i}, y_{1}^{i}\right), \ldots, d_{X_{n}}\left(x_{n}^{i}, y_{n}^{i}\right)\right) \\
\geqslant \Phi\left(d_{X_{1}}\left(x_{1}^{i}, y_{1}^{i}\right), 0, \ldots, 0\right) \geqslant \Phi(\varepsilon, 0, \ldots, 0)
\end{gathered}
$$

contrary to (3.10). Consequently id is an uniformly continuous mapping from $(P, d)$ to $\left(P, \rho_{\infty}\right)$. Condition (ii) follows.

(i) $\Rightarrow$ (ii) Let condition (ii) hold. Then by Theorem $3.5 d$ is an isotone metric product having an isotone continuation. Let $\Phi^{\circ}: \mathbb{R}_{+}^{n} \rightarrow \mathbb{R}_{+}$be an isotone function satisfying

$$
d\left(\left(x_{1}, \ldots, x_{n}\right),\left(y_{1}, \ldots, y_{n}\right)\right)=\Phi^{\circ}\left(d_{X_{1}}\left(x_{1}, y_{1}\right), \ldots, d_{X_{n}}\left(x_{n}, y_{n}\right)\right)
$$

for all $\left(x_{1}, \ldots, x_{n}\right),\left(y_{1}, \ldots, y_{n}\right) \in P$. Define the function $\Phi$ on the set $A:=D_{X_{1}} \times \cdots \times D_{X_{n}}$ as

$$
\Phi:=\left.\Phi^{\circ}\right|_{A}
$$

It is clear that $\overline{0} \in A$ and $\Phi: A \rightarrow \mathbb{R}_{+}$is isotone and amenable. In accordance with Lemma 2.7, condition (i) holds if

$$
\inf \left(\operatorname{pr}_{j}(B)\right)=0, \quad j=1, \ldots, n,
$$

for every $B \subseteq A$ with $\inf (\Phi(B))=0$ and

$$
\sup \left\{\Phi(\bar{x}): \bar{x} \in A \cap \bar{b}^{\nabla}\right\}<\infty
$$


for $\bar{b} \in \mathbb{R}_{+}^{n}$. The last inequality holds because $\Phi(\bar{x}) \leqslant \Phi^{\circ}(\bar{b})$ for every $\bar{x} \in A \cap \bar{b}^{\nabla}$. Let $B \subseteq A$ and $\inf (\Phi(B))=0$. The last equality implies that there are some sequences $\left\{\left(x_{1}^{i}, \ldots, x_{n}^{i}\right)\right\}_{i \in \mathbb{N}}$ and $\left\{\left(y_{1}^{i}, \ldots, y_{n}^{i}\right)\right\}_{i \in \mathbb{N}}$ such that $\left(d_{X_{1}}\left(x_{1}^{i}, y_{1}^{i}\right), \ldots, d_{X_{n}}\left(x_{n}^{i}, y_{n}^{i}\right)\right) \in B$ for all $i$ and

$$
\lim _{i \rightarrow \infty} d\left(\left(x_{1}^{i}, \ldots, x_{n}^{i}\right),\left(y_{1}^{i}, \ldots, y_{n}^{i}\right)\right)=0 .
$$

Since the identical mapping id from $(P, d)$ to $\left(P, \rho_{\infty}\right)$ is uniformly continuous, equality (3.13) implies

$$
\lim _{i \rightarrow \infty} \rho_{\infty}\left(\left(x_{1}^{i}, \ldots, x_{n}^{i}\right),\left(y_{1}^{i}, \ldots, y_{n}^{i}\right)\right)=0 .
$$

The last equality and the inequalities

$$
d_{X_{j}}\left(x_{j}^{i}, y_{j}^{i}\right) \leqslant \rho_{\infty}\left(\left(x_{1}^{i}, \ldots, x_{n}^{i}\right),\left(y_{1}^{i}, \ldots, y_{n}^{i}\right)\right), \quad j=1, \ldots, n,
$$

imply (3.12) for each $j \in\{1, \ldots, n\}$.

In the following theorem we show that every isotone subadditive metric product has an isotone subadditive amenable continuation.

Theorem 3.9. Let $\left(X_{1}, d_{X_{1}}\right), \ldots,\left(X_{n}, d_{X_{n}}\right)$ be nonempty metric spaces. The following conditions are equivalent for every metric $d$ on $P=X_{1} \times \cdots \times X_{n}$.

(i) There is an isotone metric preserving function $\Psi: \mathbb{R}_{+}^{n} \rightarrow \mathbb{R}_{+}$such that

$$
d\left(\left(x_{1}, \ldots, x_{n}\right),\left(y_{1}, \ldots, y_{n}\right)\right)=\Psi\left(d_{X_{1}}\left(x_{1}, y_{1}\right), \ldots, d_{X_{n}}\left(x_{n}, y_{n}\right)\right)
$$

for all $\left(x_{1}, \ldots, x_{n}\right),\left(y_{1}, \ldots, y_{n}\right) \in P$.

(ii) $d$ is an isotone subadditive metric product.

Proof. The implication (i) $\Rightarrow$ (ii) is almost evident (see Remark 2.10).

Suppose condition (ii) holds. Then there is an isotone subadditive function $\Phi: A \rightarrow \mathbb{R}_{+}$such that

$$
d\left(\left(x_{1}, \ldots, x_{n}\right),\left(y_{1}, \ldots, y_{n}\right)\right)=\Phi\left(d_{X_{1}}\left(x_{1}, y_{1}\right), \ldots, d_{X_{n}}\left(x_{n}, y_{n}\right)\right)
$$

for all $\left(x_{1}, \ldots, x_{n}\right),\left(y_{1}, \ldots, y_{n}\right) \in P$ where $A=D_{X_{1}} \times \cdots \times D_{X_{n}}$. Let

$$
J_{0}:=\left\{j \in\{1, \ldots, n\}: \operatorname{card} X_{j}=1\right\} .
$$

It is clear that $j \in J_{0}$ if and only if $\operatorname{pr}_{j}(\bar{a})=0$ for every $\bar{a} \in A$. Let $\bar{e}^{1}=(1,0, \ldots, 0), \bar{e}^{2}=(0,1, \ldots, 0), \ldots, \bar{e}^{n}=(0,0, \ldots, 1)$ be the standard basis 
vectors in $\mathbb{R}^{n}$. Denote by $B$ the Minkowski sum of $A$ and of the convex cone generated by the set $\left\{\bar{e}^{j}: j \in J_{0}\right\}$, i.e., $\bar{x} \in B$ if and only if there are $\alpha_{j} \geqslant 0, j \in J_{0}$, and $\bar{a} \in A$ such that

$$
\bar{x}=\bar{a}+\sum_{j \in J_{0}} \alpha_{j} \bar{e}^{j}
$$

It is clear that $A \subseteq B$. Since the linear space $\mathbb{R}^{n}$ is a direct sum of linear spaces generating by the vectors $\bar{e}^{j}, j \in J_{0}$, and by the set $A$, the equality

$$
\bar{a}+\sum_{j \in J_{0}} \alpha_{j} \bar{e}^{j}=\bar{b}+\sum_{j \in J_{0}} \beta_{j} \bar{e}^{j}
$$

with $\bar{a}, \bar{b} \in A$ implies $\bar{a}=\bar{b}$ and $\sum_{j \in J_{0}} \alpha_{j} \bar{e}^{j}=\sum_{j \in J_{0}} \beta_{j} \bar{e}^{j}$. Let $c$ be a strictly positive constant. Define a function $\Phi^{\circ}: B \rightarrow \mathbb{R}_{+}$by the rule: if $\bar{x} \in B$ has a representation (3.15), then

$$
\Phi^{\circ}(\bar{x})= \begin{cases}\Phi(\bar{a}) & \text { if } \sum_{j \in J_{0}} \alpha_{j} \bar{e}^{j}=0 ; \\ \Phi(\bar{a})+c & \text { if } \sum_{j \in J_{0}} \alpha_{j} \bar{e}^{j} \neq 0 .\end{cases}
$$

The uniqueness of representation (3.15) implies that $\Phi^{\circ}$ is correctly defined and, moreover, from (3.16) it follows that $\left.\Phi^{\circ}\right|_{A}=\Phi$. As in the second part of the proof of Lemma 2.11 we can show that $\Phi^{\circ}$ is isotone and subadditive. Moreover it is easy to see that the set $\mathbf{S}(\bar{x})=\mathbf{S}(\bar{x}, B)$, defined as in the proof of Lemma 2.11, is nonempty. Consequently, the function $\Psi: \mathbb{R}_{+}^{n} \rightarrow \mathbb{R}_{+}$,

$$
\Psi(\bar{x})=\inf \left\{\sum_{i=1}^{m} \bar{x}^{i}:\left(\bar{x}^{1}, \ldots, \bar{x}^{m}\right) \in \overline{\mathbf{S}}(\bar{x}, B)\right\},
$$

(cf. (2.20) ) is an isotone subadditive continuation of $\Phi^{\circ}$. Thus (3.14) holds for all $\left(x_{1}, \ldots, x_{n}\right),\left(y_{1}, \ldots, y_{n}\right) \in P$. Now to prove $(i)$ it is sufficient to show that $\Psi$ is amenable. Let us do it.

Equality (3.16) and $\left.\Psi\right|_{A}=\left.\Phi^{\circ}\right|_{A}=\Phi$ imply that $\Psi(\overline{0})=0$. Let $\bar{x}=$ $\left(x_{1}, \ldots, x_{n}\right)$ be a point of $\mathbb{R}_{+}^{n}$ such that $\Psi(\bar{x})=0$. We shall show that $x_{j}=\operatorname{pr}_{j}(\bar{x})=0$ for every $j=1, \ldots, n$. Suppose there is $j_{0} \in J_{0}$ such that $x_{j_{0}}>0$. Since $\bar{x} \geqslant \operatorname{pr}_{j_{0}}(\bar{x}) \bar{e}^{j_{0}}$ and $\Psi$ is isotone, we have

$$
\Psi(\bar{x}) \geqslant \Psi\left(\operatorname{pr}_{j_{0}}(\bar{x}) \bar{e}^{j_{0}}\right)=\Phi^{\circ}\left(\operatorname{pr}_{j_{0}}(\bar{x}) \bar{e}^{j_{0}}\right)=c>0 .
$$

Hence if $\operatorname{pr}_{j_{0}}(\bar{x})>0$, then $j_{0} \in\{1, \ldots, n\} \backslash J_{0}$. The membership $j_{0} \in$ $\{1, \ldots, n\} \backslash J_{0}$ and the definition of $J_{0}$ imply that card $X_{j_{0}} \geqslant 2$. Consequently 
there are $z_{j_{0}}, y_{j_{0}} \in X_{j_{0}}$ such that $d_{X_{j_{0}}}\left(z_{j_{0}}, y_{j_{0}}\right)>0$. Hence there is $m \in \mathbb{N}$ for which $d_{X_{j_{0}}}\left(z_{j_{0}}, y_{j_{0}}\right) \leqslant m \operatorname{pr}_{j_{0}}(\bar{x})$ so that

$$
d_{X_{j_{0}}}\left(z_{j_{0}}, y_{j_{0}}\right) \bar{e}^{j_{0}} \leqslant m \operatorname{pr}_{j_{0}}(\bar{x}) \bar{e}^{j_{0}} .
$$

It is easy to see that $d_{X_{j_{0}}}\left(z_{j_{0}}, y_{j_{0}}\right) \bar{e}^{j_{0}} \in A$. Since $\Psi$ is isotone and subadditive, inequality (3.17) implies

$$
\begin{gathered}
\Phi\left(d_{X_{j_{0}}}\left(z_{j_{0}}, y_{j_{0}}\right) \bar{e}^{j_{0}}\right)=\Psi\left(d_{X_{j_{0}}}\left(z_{j_{0}}, y_{j_{0}}\right) \bar{e}^{j_{0}}\right) \leqslant \Psi\left(m \operatorname{pr}_{j_{0}}(\bar{x}) \bar{e}^{j_{0}}\right) \\
\leqslant m \Psi\left(\operatorname{pr}_{j_{0}}(\bar{x}) \bar{e}^{j_{0}}\right) \leqslant m \Psi(\bar{x})=0 .
\end{gathered}
$$

Hence $\Phi$ is not amenable, contrary to the definition. Thus if $\Psi(\bar{x})=0$ and $\operatorname{pr}_{j}(\bar{x})>0$, then $j \notin J_{0}$ and $j \notin\{1, \ldots, n\} \backslash J_{0}$, i.e., the implication $\Psi(\bar{x}=0) \Rightarrow(\bar{x}=0)$ holds. The function $\Psi$ is amenable, as required.

Theorems 3.9 and 3.8 imply the following

Corollary 3.10. Let $\left(X_{1}, d_{X_{1}}\right), \ldots,\left(X_{n}, d_{X_{n}}\right)$ be nonempty metric spaces. If $d$ is an isotone subadditive metric product on $P$, then the identical mapping

$$
\operatorname{id}\left(x_{1}, \ldots, x_{n}\right)=\left(x_{1}, \ldots, x_{n}\right), \quad\left(x_{1}, \ldots, x_{n}\right) \in P,
$$

is bornologous as a mapping from $\left(P, \rho_{\infty}\right)$ to $(P, d)$ and uniformly continuous as a mapping from $(P, d)$ to $\left(P, \rho_{\infty}\right)$.

\section{Modulus of continuity of bornologous functions}

We shall say that a function $f: \mathbb{R}_{+}^{n} \rightarrow \mathbb{R}_{+}$is nonconstant with respect to the variable $x_{1}$ if there exist nonnegative numbers $a_{i}, i=2, \ldots, n$, such that the function $f\left(x_{1}, a_{2}, \ldots, a_{n}\right)$ is nonconstant as a function of one variable $x_{1}$. Analogously we define the functions $f: \mathbb{R}_{+}^{n} \rightarrow \mathbb{R}_{+}$which are nonconstant w.r.t. the variable $x_{i}$ for $2 \leqslant i \leqslant n$.

Let us denote by $\mathcal{W}$ the family all bornologous functions $g: \mathbb{R}_{+}^{n} \rightarrow \mathbb{R}_{+}$ which are nonconstant w.r.t. every variable.

It is easy to prove that every uniformly continuous function $g: \mathbb{R}_{+}^{n} \rightarrow \mathbb{R}_{+}$ which is nonconstant w.r.t. all variables belongs to $\mathcal{W}$ (cf. Example 3.6) .

For $g \in \mathcal{W}$ we define the "modulus of continuity" as follows

$$
\omega(g, \bar{\varepsilon}):=\sup _{\substack{|\bar{x}-\bar{y}| \leqslant \bar{\varepsilon} \\ \bar{x}, \bar{y} \in \mathbb{R}_{+}^{n}}}|g(\bar{x})-g(\bar{y})|
$$

where $\bar{\varepsilon}=\left(\varepsilon_{1}, \ldots, \varepsilon_{n}\right) \in \mathbb{R}_{+}^{n}$ and the inequality $|\bar{x}-\bar{y}| \leqslant \bar{\varepsilon}$ is understood in accordance with (2.1). 
Remark 4.1. For $\bar{\varepsilon}=\overline{0}$ formula (4.1) gives us the equality $\omega(\bar{g}, \overline{0})=0$.

It was noted in [16] that the inequality

$$
|f(a)-f(b)| \leqslant f(|a-b|)
$$

holds for every metric preserving function $f: \mathbb{R}_{+} \rightarrow \mathbb{R}_{+}$and all $a, b \in \mathbb{R}_{+}$. In the following lemma we give a multivariate version of this inequality for isotone metric preserving functions of several variables.

Lemma 4.2. Let $F \in \mathfrak{F}_{i}^{n}$. The inequality

$$
\mid F(\bar{x})-F(\bar{y})) \mid \leqslant F(|\bar{x}-\bar{y}|)
$$

holds for all $\bar{x}, \bar{y} \in \mathbb{R}_{+}^{n}$ with $\left.|\bar{x}-\bar{y}|=\left(\left|x_{1}-y_{1}\right|, \ldots, \mid x_{n}-y_{n}\right) \mid\right)$.

Proof. Let $\bar{x}, \bar{y} \in \mathbb{R}_{+}^{n}$. We may assume, without loss of generality, that

$$
F(\bar{x}) \geqslant F(\bar{y})
$$

It is easy to see that

$$
\bar{x} \leqslant \bar{y}+|\bar{x}-\bar{y}| .
$$

Since $F$ is isotone and subadditive (see Theorem 3.1), inequalities (4.3) and (4.4) imply (4.2),

$$
\begin{gathered}
|F(\bar{x})-F(\bar{y})|=F(\bar{x})-F(\bar{y}) \leqslant F(\bar{y}+|\bar{x}-\bar{y}|)-F(\bar{y}) \\
\leqslant F(\bar{y})+F(|\bar{x}-\bar{y}|)-F(\bar{y})=F(|\bar{x}-\bar{y}|) .
\end{gathered}
$$

Theorem 4.3. Let $F$ be an arbitrary nonnegative function defined on $\mathbb{R}_{+}^{n}$. Then $F$ belongs to $\mathfrak{F}_{i}^{n}$ if and only if there exists $g \in \mathcal{W}$ such that

$$
F(\bar{\varepsilon})=\omega(g, \bar{\varepsilon})
$$

for every $\bar{\varepsilon} \in \mathbb{R}_{+}^{n}$.

Proof. Suppose $F \in \mathfrak{F}_{i}^{n}$. Let us prove that there exists $g \in \mathcal{W}$ such that equality (4.5) holds for every $\bar{\varepsilon} \in \mathbb{R}_{+}^{n}$. It suffices to show that

$$
F \in \mathcal{W}
$$

and

$$
F(\bar{\varepsilon})=\omega(F, \bar{\varepsilon})
$$


Let us verify that $F$ is nonconstant w.r.t. each variable. Indeed, since $F$ is amenable we have

$$
0=F(0, \ldots, 0) \neq F(t, 0, \ldots, 0) \text { if } t>0 .
$$

Thus, $F$ is nonconstant w.r.t. the first variable. Similarly we can establish that $F$ is nonconstant w.r.t. other variables. Thus, to prove (4.6) it remains to verify that for every $\varepsilon>0$ there exists $\delta(\varepsilon)>0$ such that for every $\bar{x}=\left(x_{1}, \ldots, x_{n}\right), \bar{y}=\left(y_{1}, \ldots, y_{n}\right) \in \mathbb{R}_{+}^{n}$ the inequality

$$
\sum_{i=1}^{n}\left|x_{i}-y_{i}\right|<\varepsilon
$$

implies the inequality

$$
|F(\bar{x})-F(\bar{y})| \leqslant \delta(\varepsilon) .
$$

Suppose that inequality (4.8) holds. By Lemma 4.2 we obtain

$$
|F(\bar{x})-F(\bar{y})| \leqslant F(|\bar{x}-\bar{y}|) .
$$

Inequality (4.8) implies that $|\bar{x}-\bar{y}| \leqslant \bar{\varepsilon}$ where $\bar{\varepsilon}=(\varepsilon, \ldots, \varepsilon) \in \mathbb{R}_{+}^{n}$. Consequently we obtain $F(|\bar{x}-\bar{y}|) \leqslant F(\bar{\varepsilon})$. Thus inequality (4.9) holds with $\delta(\varepsilon)=F(\bar{\varepsilon})$. It still remains to note that equality (4.7) follows directly from the definition of modulus of continuity and Lemma 4.2.

Suppose $g \in \mathcal{W}$ and equality (4.5) holds for every $\bar{\varepsilon} \in \mathbb{R}_{+}^{n}$. It is necessary to show that $F \in \mathfrak{F}_{i}^{n}$. Note that monotonicity and nonnegativity $F$ follow directly from (4.1). Let us show that $F(\bar{\varepsilon})>0$ if $\bar{\varepsilon}>\overline{0}$. By Remark 4.1 we have $F(\overline{0})=0$

The inequality $\bar{\varepsilon}>\overline{0}$ implies that there exists $i_{0} \in\{1, \ldots, n\}$ such that $\varepsilon_{i_{0}}>0$. For convenience we may consider the case when $i_{0}=1$. In (4.5) the function $g$ belongs to $\mathcal{W}$. Consequently $g$ is nonconstant w.r.t. all variables. Hence there are some numbers $a_{i} \in \mathbb{R}_{+}, i=2, \ldots, n$, such that the function

$$
g^{*}(t):=g\left(t, a_{2}, \ldots, a_{n}\right), \quad t \in \mathbb{R}_{+},
$$

is nonconstant. Suppose $F(\varepsilon)=0$. Then we obtain $g^{*}\left(t_{1}\right)=g^{*}\left(t_{2}\right)$ whenever $\left|t_{2}-t_{1}\right| \leqslant \varepsilon_{1}$. Consequently $g^{*}(t)=$ constant. This contradiction implies the desired inequality $F(\bar{\varepsilon})>0$ for $\bar{\varepsilon}>\overline{0}$.

It remains to verify that

$$
F(\bar{x}+\bar{y}) \leqslant F(\bar{x})+F(\bar{y})
$$

for $\bar{x}, \bar{y} \in \mathbb{R}_{n}^{+}$. To prove (4.10) consider $\bar{u}, \bar{v} \in \mathbb{R}_{+}^{n}$ for which $|\bar{u}-\bar{v}| \leqslant \bar{x}+\bar{y}$. The simple geometrical reasoning shows that there exists $\bar{w} \in \mathbb{R}_{+}^{n}$ such that

$$
|\bar{u}-\bar{w}| \leqslant \bar{x} \text { and }|\bar{w}-\bar{v}| \leqslant \bar{y} .
$$


Now using (4.1) and the two inequalities given above we get

$|g(\bar{u})-g(\bar{v})| \leqslant|g(\bar{u})-g(\bar{w})|+|g(\bar{w})-g(\bar{v})| \leqslant \omega(g, \bar{x})+\omega(g, \bar{y})=F(\bar{x})+F(\bar{y})$.

From the other hand

$$
F(\bar{x}+\bar{y})=\omega(g, \bar{x}+\bar{y})=\sup _{\substack{|\bar{u}-\bar{v}| \leqslant \bar{x}+\bar{y} \\ \bar{x}, \bar{y} \in \mathbb{R}_{+}^{n}}}|g(\bar{u})-g(\bar{v})|,
$$

which proves (4.10). Now the membership relation $F \in \mathfrak{F}_{i}^{n}$ follows from Theorem 3.1.

The first part of the proof of Theorem 4.3 shows that $\mathfrak{F}_{i}^{n} \subseteq \mathcal{W}$. The following selects the metric preserving functions from the functions belonging to $\mathcal{W}$.

Corollary 4.4. The set $\mathfrak{F}_{i}^{n}$ coincides with the set of fixed points of the mapping

$$
\mathcal{W} \ni g \mapsto \omega(g, \cdot) \in \mathcal{W}
$$

where $\omega(g, \cdot)$ is defined by (4.1).

\section{Some 3l-universal subspaces of $\mathbb{R}$}

We shall say that a metric space $(X, d)$ is $3 l$-universal (linear three universal) if and only if every three-point metric subspace of the real line is isometrically embeddable in $(X, d)$, i.e., for all $a, b \in \mathbb{R}_{+}$there are $x_{1}, x_{2}, x_{3} \in X$ such that

$$
d\left(x_{1}, x_{2}\right)=a, \quad d\left(x_{2}, x_{3}\right)=b, \quad d\left(x_{1}, x_{3}\right)=a+b .
$$

The following theorem gives a set of sufficient and necessary conditions under which an isotone function $\Phi: \mathbb{R}_{+}^{n} \rightarrow \mathbb{R}_{+}$belongs to $\mathfrak{F}_{i}^{n}$.

Theorem 5.1. Let $\Phi: \mathbb{R}_{+}^{n} \rightarrow \mathbb{R}_{+}$be an isotone function. The following conditions are equivalent.

(i) The function $\Phi\left(d_{X_{1}}(\cdot, \cdot), \ldots, d_{X_{n}}(\cdot, \cdot)\right)$ is a metric on $P=X_{1} \times \cdots \times X_{n}$ for all metric spaces $\left(X_{1}, d_{X_{1}}\right), \ldots,\left(X_{n}, d_{X_{n}}\right)$.

(ii) The function $\Phi\left(d_{X_{1}}(\cdot, \cdot), \ldots, d_{X_{n}}(\cdot, \cdot)\right)$ is a metric on $P=\mathbb{R}^{n}$ with $X_{1}=\cdots=X_{n}=\mathbb{R}$,

$$
d_{X_{1}}\left(x_{1}, y_{1}\right)=\left|x_{1}-y_{1}\right|, \ldots, d_{X_{n}}\left(x_{n}, y_{n}\right)=\left|x_{n}-y_{n}\right| .
$$


(iii) The function $\Phi\left(d_{X_{1}}(\cdot, \cdot), \ldots, d_{X_{n}}(\cdot, \cdot)\right)$ is a metric on $P=X_{1} \times \cdots \times X_{n}$ for all three-point subspaces $X_{1}, \ldots, X_{n}$ of $\mathbb{R}$ with the usual metric $d(x, y)=|x-y|$.

(iv) There are 3 l-universal metric spaces $\left(X_{1}, d_{X_{1}}\right), \ldots,\left(X_{n}, d_{X_{n}}\right)$ such that $\Phi\left(d_{X_{1}}(\cdot, \cdot), \ldots, d_{X_{n}}(\cdot, \cdot)\right)$ is a metric on $P=X_{1} \times \cdots \times X_{n}$.

Sketch of the proof. Condition (i) is a reformulation of the definition of the set $\mathfrak{F}_{i}^{n}$. The implications (i) $\Rightarrow$ (ii), (i) $\Rightarrow$ (iii), (i) $\Rightarrow($ iv) are evident. Each condition from (ii), (iii), (iv) implies that for every $\left(a_{1}, \ldots, a_{n}\right),\left(b_{1}, \ldots, b_{n}\right) \in$ $\mathbb{R}_{+}^{n}$ there are triangles $\left\{x_{i}, y_{i}, z_{i}\right\} \subseteq X_{i}$ such that

$$
d_{X_{i}}\left(x_{i}, y_{i}\right)=a_{i}, \quad d_{X_{i}}\left(y_{i}, z_{i}\right)=b_{i}, \quad d_{X_{i}}\left(x_{i}, z_{i}\right)=a_{i}+b_{i}, \quad i=1, \ldots, n .
$$

Since $\Phi\left(d_{X_{1}}(\cdot, \cdot), \ldots, d_{X_{n}}(\cdot, \cdot)\right)$ is a metric on $P$, the triangle inequality implies

$$
\Phi\left(a_{1}+b_{1}, \ldots, a_{n}+b_{n}\right) \leqslant \Phi\left(a_{1}, \ldots, a_{n}\right)+\Phi\left(b_{1}, \ldots, b_{n}\right) .
$$

Hence $\Phi$ is subadditive. The implications (ii) $\Rightarrow($ i), (iii) $\Rightarrow($ i), (iv) $\Rightarrow($ i) follow from Theorem 3.1

Remark 5.2. Theorem 5.1 has a natural analog for arbitrary metric preserving functions $\Phi: \mathbb{R}_{+}^{n} \rightarrow \mathbb{R}_{+}$. For example, Theorem 2.1 in [7] or Lemma 1 in [1] or Lemma 7 in Section 5 of [2] are similar to the equivalence (i) $\Leftrightarrow$ (iii) from Theorem 5.1

Let us consider some $3 l$-universal metric subspaces of $\mathbb{R}=(-\infty, \infty)$.

The set $\mathbb{R}$ with the usual addition and the multiplication on rational numbers forms a vector space over the field $\mathbb{Q}$. If $X$ is a linear subspace of $\mathbb{R}$ and $B \subseteq X$, then $B$ is called a Hamel basis of $X$ if and only if every nonzero $x \in X$ can be uniquely represented as a finite linear combination

$$
x=\sum_{i=1}^{n} r_{i} b_{i}
$$

where $b_{1}, \ldots, b_{n}$ are distinct elements of $B$ and $r_{1}, \ldots, r_{n} \in \mathbb{Q} \backslash\{0\}$. It is well known and easy to prove by transfinite induction that such basis exists for every linear subspace of $\mathbb{R}$. In particular $\varnothing$ is a Hamel basis for $X=\{0\}$.

Proposition 5.3. Let $X$ be a linear subspace of $\mathbb{R}$. If $\mathbb{R} \backslash X \neq \varnothing$, then the set $\mathbb{R} \backslash X$ is 3l-universal. 
Proof. Let $X \neq \mathbb{R}$. It is sufficient to show that for every $a, b \in \mathbb{R}_{+}$there are $x_{0}, y_{0}, z_{0} \in \mathbb{R} \backslash X$ such that

$$
x_{0}-y_{0}=a \text { and } y_{0}-z_{0}=b .
$$

This is evident for $X=\{0\}$. Suppose that $X \neq\{0\}$ and that $B$ is a Hamel basis of $X$. Let $x^{*}, y^{*}$ and $z^{*}$ be some nonzero points of $\mathbb{R}$ such that

$$
x^{*}-y^{*}=a \text { and } y^{*}-z^{*}=b .
$$

Since $B$ is a rationally independent set, there is a Hamel basis $B_{1}$ of $\mathbb{R}$ such that $B_{1} \supseteq B$. The numbers $x^{*}, y^{*}, z^{*}$ can be uniquely represented as

$$
x^{*}=\sum_{i=1}^{n(x)} r_{i}(x) b_{i}(x), \quad y^{*}=\sum_{i=1}^{n(y)} r_{i}(y) b_{i}(y) \text { and } z^{*}=\sum_{i=1}^{n(z)} r_{i}(z) b_{i}(z)
$$

with $r_{i}(x), r_{i}(y), r_{i}(z) \in \mathbb{Q} \backslash\{0\}$ and $b_{i}(x), b_{i}(y), b_{i}(z) \in B_{1}$. Since

$$
\mathbb{R} \supseteq X \neq \mathbb{R}
$$

the set $B_{1} \backslash B$ is nonempty. Let $b_{0} \in B_{1} \backslash B$. Define $x_{0}, y_{0}$ and $z_{0}$ as

$$
x_{0}=x^{*}+\left(r_{0}+1\right) b_{0}, \quad y_{0}=y^{*}+\left(r_{0}+1\right) b_{0}, \quad z_{0}=z^{*}+\left(r_{0}+1\right) b_{0}
$$

where

$$
r_{0}:=\max \left\{\bigvee_{i=1}^{n(x)}\left|r_{i}(x)\right|, \bigvee_{i=1}^{n(y)}\left|r_{i}(y)\right|, \bigvee_{i=1}^{n(z)}\left|r_{i}(z)\right|\right\}
$$

The numbers $x_{0}, y_{0}, z_{0}$ can be represented as finite rational nonzero linear combinations of elements of $B_{1}$. The uniqueness of such representations and equalities (5.4), (5.5), (5.6) imply that $x_{0}, y_{0}, z_{0} \in \mathbb{R} \backslash X$. Moreover (5.2) follows from (5.3) and (5.5).

Corollary 5.4. Let $X$ be a subfield of the field $\mathbb{R}$. If $\mathbb{R} \backslash X \neq \varnothing$, then $\mathbb{R} \backslash X$ is 3l-universal.

Proof. Every field $X \subseteq \mathbb{R}$ can be regarded as a linear space over the field $\mathbb{Q}$ of rational numbers.

Corollary 5.5. The set $T$ of all real transcendental numbers is 3 -universal

Proof. $\mathbb{R} \backslash T$ is the set of all real algebraic numbers. It is well known that this is a subfield of the field $\mathbb{R}$. 
Proposition 5.6. Let $X$ be a linear subspace of $\mathbb{R}$. Then $X$ is 3 -universal if and only if $X=\mathbb{R}$.

Proof. It is clear that $\mathbb{R}$ is $3 l$-universal. Suppose that $X \neq \mathbb{R}$. Let $a$ be a positive number from $\mathbb{R} \backslash X$. If $X$ is $3 l$-universal, then there is $x, y \in X$ such that $|x-y|=a$. We may assume that $x \geqslant y$. Hence we obtain the contradiction $X \ni(x-y)=|x-y| \notin X$.

A simple modification of the proof of Proposition 5.3 shows that the implication

- if $\mathbb{R} \backslash X \neq \varnothing$, then there is an isometric embedding $f: A \rightarrow \mathbb{R} \backslash X$

holds for every finite subset $A$ of $\mathbb{R}$ and every linear subspace $X$ of $\mathbb{R}$. In particular, we obtain the next interesting generalization of Corollary 5.5 .

Corollary 5.7. Let $A$ be a finite subset of $\mathbb{R}$. Then there is an isometric embedding of $A$ in the set $T$ of all real transcendental numbers.

In the following example we construct a set $X \subseteq \mathbb{R}_{+}$such that the distance set $D_{X}=\{|x-y|: x, y \in X\}$ is the same as $\mathbb{R}_{+}$but $X$ is not $3 l$ universal. To this end, we use the triadic Cantor set. Recall the definition. Let $x \in[0,1]$ and expand $x$ as

$$
x=\sum_{n=1}^{\infty} \frac{b_{n}(x)}{3^{n}}, \quad b_{n}(x) \in\{0,1,2\} .
$$

The Cantor set $C$ is the set of points from $[0,1]$ which have expansion (5.7) using only the digits 0 and 2. Thus $x \in C$ if and only if $x$ has a triadic representation

$$
x=\sum_{n=1}^{\infty} \frac{2 \alpha_{m}}{3^{m}}
$$

where $\alpha_{m}=\alpha_{m}(x) \in\{0,1\}$.

Example 5.8. Define a set $C^{e}$ as

$$
C^{e}=\bigcup_{n=0}^{\infty} 3^{n} C
$$

where $3^{n} C=\left\{3^{n} x: x \in C\right\}$. It follows from (5.8) that a real number $t$ belongs to $C^{e}$ if and only if $t$ has a base 3 expansion with the digits 0 and 2 only, i.e.,

$$
t=\sum_{j=-\infty}^{M(t)} a_{j}(t) 3^{j}
$$


where $M(t) \in \mathbb{Z}$ and $a_{j}(t) \in\{0,2\}$. It is easy to prove (see, for example, [19, Chapter 8, Example 3]) that the distance set of the triadic Cantor set $C$ is $[0,1], D_{C}=[0,1]$. Consequently we obtain $D_{3^{n} C}=\left[0,3^{n}\right]$ for every natural number $n$. Hence we have $\mathbb{R}_{+} \supseteq D_{C^{e}} \supseteq \bigcup_{n=0}^{\infty}\left[0,3^{n}\right]=\mathbb{R}_{+}$, so that $D_{C^{e}}=\mathbb{R}_{+}$.

We claim that there are no points $x, y, z \in C^{e}$ such that

$$
|x-y|=\frac{1}{3} \text { and }|x-z|=\frac{1}{6}
$$

Suppose contrary that $x, y, z \in C^{e}$ and satisfy (5.10). Let $j_{0}$ be the largest index $j$ such that $a_{j}(x) \neq a_{j}(y)$ where $a_{j}(x)$ and $a_{j}(y)$ are the coefficients from expansion (5.9) for $x$ and, respectively, $y$. Then we obtain

$$
\begin{gathered}
\frac{1}{3}=|x-y| \geqslant 2 \cdot 3^{j_{0}}-\left|\sum_{j=-\infty}^{j_{0}-1}\left(a_{j}(x)-a_{j}(y)\right) 3^{j}\right| \geqslant 2 \cdot 3^{j_{0}}-\sum_{j=-\infty}^{j_{0}-1}\left|a_{j}(x)-a_{j}(y)\right| 3^{j} \\
\geqslant 2 \cdot 3^{j_{0}}-\sum_{j=-\infty}^{j_{0}-1} 2 \cdot 3^{j}=2 \cdot 3^{j_{0}}-3 \cdot 3^{j_{0}-1}=3^{j_{0}} .
\end{gathered}
$$

Consequently the inequality $j_{0} \leqslant-1$ holds. Hence the equality

$$
a_{j}(x)=a_{j}(y)
$$

holds for all $j \geqslant 0$. Similarly we have

$$
a_{j}(x)=a_{j}(z)
$$

for all $j \geqslant 0$. Equations (5.11) and (5.12) imply that there is a constant $c_{1} \geqslant 0$ such that

$$
c_{1}=\sum_{j=0}^{M(x)} a_{j}(x) 3^{j}=\sum_{j=0}^{M(y)} a_{j}(y) 3^{j}=\sum_{j=0}^{M(z)} a_{j}(z) 3^{j} .
$$

Define the points $x^{*}, y^{*}, z^{*}$ as $x^{*}=x-c_{1}, y^{*}=y-c_{1}$ and $z^{*}=z-c_{1}$. Then the equalities $\left|x^{*}-y^{*}\right|=\frac{1}{3}$ and $\left|x^{*}-z^{*}\right|=\frac{1}{6}$ hold and $x^{*}, y^{*}, z^{*} \in C$. Since $\left(\frac{1}{3}, \frac{2}{3}\right) \cap C=\varnothing$ and $C \subseteq[0,1]$, the equality $\left|x^{*}-y^{*}\right|=\frac{1}{3}$ shows that the following six combinations
$\left(i_{1}\right) \quad x^{*}=0, y^{*}=\frac{1}{3}$
$\left(i_{2}\right) \quad x^{*}=\frac{1}{3}, y^{*}=\frac{2}{3}$,
$\left(i_{3}\right) \quad x^{*}=\frac{2}{3}, y^{*}=1$,
$\left(i_{4}\right) \quad x^{*}=\frac{1}{3}, y^{*}=0$,
$\left(i_{5}\right) \quad x^{*}=\frac{2}{3}, y^{*}=\frac{1}{3}$,
$\left(i_{6}\right) \quad x^{*}=1, y^{*}=\frac{2}{3}$ 
are the only possible. This equality and the next one $\left|x^{*}-z^{*}\right|=\frac{1}{6}$ give us the membership relation

$$
z^{*} \in\left\{-\frac{1}{6}, \frac{1}{6}, \frac{1}{2}, \frac{5}{6}, \frac{7}{6}\right\} .
$$

Since $C \cap\left\{-\frac{1}{6}, \frac{1}{6}, \frac{1}{2}, \frac{5}{6}, \frac{7}{6}\right\}=\varnothing$ we obtain that $z^{*} \notin C$, contrary to the supposition $x^{*}, y^{*}, z^{*} \in C$. Thus $C^{e}$ is not $3 l$-universal.

Remark 5.9. $C^{e}$ is the smallest (w.r.t. the relation $\subseteq$ ) set $X \subseteq \mathbb{R}_{+}$satisfying the conditions $C \subseteq X$ and $3 X=X$.

\section{References}

[1] A. Berning, T. Foertsch, and V. Schroeder, Non standart metric products// Beitr. Algebra Geom., 44, (2003), 499-510.

[2] T. Foertsch and V. Schroeder, Minkovski-versus Eucledean rank for products of metric spaces// Adv. Geom., 2, (2002), 123-131.

[3] M. Moszyìska, On the uniqueness problem for metric products// Glasnik Matematićki, 27(47), (1992), 145-158.

[4] C. Tardif, Prefibers and the cartesian product of metric spaces// Discrete Mathemetics, 109, (1992), 283-288.

[5] S. Avgustinovich and D. Fon-Der-Flaass, Cartesian products of graphs and metric spaces// Europ. J. Combinatorics, 21, (2000), 847-851.

[6] F. Terpe, Metric preserving functions of several variables// Proc. Conf. Topology and Measure V Greifswald (1988), 169-174.

[7] I. Herburt and M. Moszyńska, On metric products// Coll. Math., 62, (1991), 121-133.

[8] J. Olędzki and S. Spież, Remarks on intrinsic isometries// Fund. Math., 119, (1983), 241-247.

[9] O. Dovgoshey and O. Martio, Products of metric spaces, covering numbers, packing numbers, and characterizations of ultrametric spaces// Rev. Roumaine Math. Pures Appl., 54, (2009), N5-6, 423-439.

[10] J. Borsik and J. Doboř, On a product of metric spaces// Math. Slovaca 31, N2, (1981), 193-205. 
[11] P. Corazza, Introduction to metric preserving functions//Amer. Math. Monthly, 104, N4, (1999) 309-323.

[12] J. Dobǒs, A survey on metric preserving functions//Q \& A in General Topology 13, N4, (1995), 129-134.

[13] T.K. Sreenivasan, Some properties of distance functions// J. Indian Math. Soc. (N.S.) 11, (1947), 38-43.

[14] F. Terpe, Metric preserving functions// Proc. Conf. Topology and Measure IV Greifswald (1984), 189-197.

[15] J. Dobǒs, Metric preserving functions, Košce, Slovak Republic, 1998.

[16] J. Borsik and J. Doboš, On metric preserving functions// Real. Anal. Exh. 13, (1987-1988), 285-293.

[17] O. Dovgoshey and O. Martio, Functions transferring metrics to metrics// Beitr. Algebra Geom. DOI 10.1007/s 13336-011-0061-7.

[18] J. Roe, Lectures on coarse geometry, University lecture series, 31, American Mathematical Society, Providence, RI, 2003.

[19] B. Gelbaum and J. Olmsted, Counterexamples in analysis, The Mathesis Series Holden-Day, Inc., San Francisco, Calif.-London-Amsterdam, 1964.

Oleksiy Dovgoshey

Institute of Applied Mathematics and Mechanics of NASU, R. Luxemburg Str. 74, Donetsk 83114, Ukraine

E-mail: aleksdov@mail.ru

\section{Evgeniy Petrov}

Institute of Applied Mathematics and Mechanics of NASU, R. Luxemburg Str. 74, Donetsk 83114, Ukraine

E-mail: eugeniy.petrov@gmail.com

\section{Galina Kozub}

Donetsk National University, Universitetska Str. 24, Donetsk 83055, Ukraine

E-mail: kozub_galina@mail.ru 